\title{
Mobile Phone Apps for Quality of Life and Well-Being Assessment in Breast and Prostate Cancer Patients: Systematic Review
}

Esther Rincon $^{1^{*}}, \mathrm{PhD}$; Francisco Monteiro-Guerra ${ }^{2^{*}}, \mathrm{MS}$; Octavio Rivera-Romero ${ }^{3}, \mathrm{PhD}$; Enrique Dorronzoro-Zubiete ${ }^{3}$, $\mathrm{PhD}$; Carlos Luis Sanchez-Bocanegra ${ }^{4}, \mathrm{PhD}$; Elia Gabarron ${ }^{5}, \mathrm{PhD}$

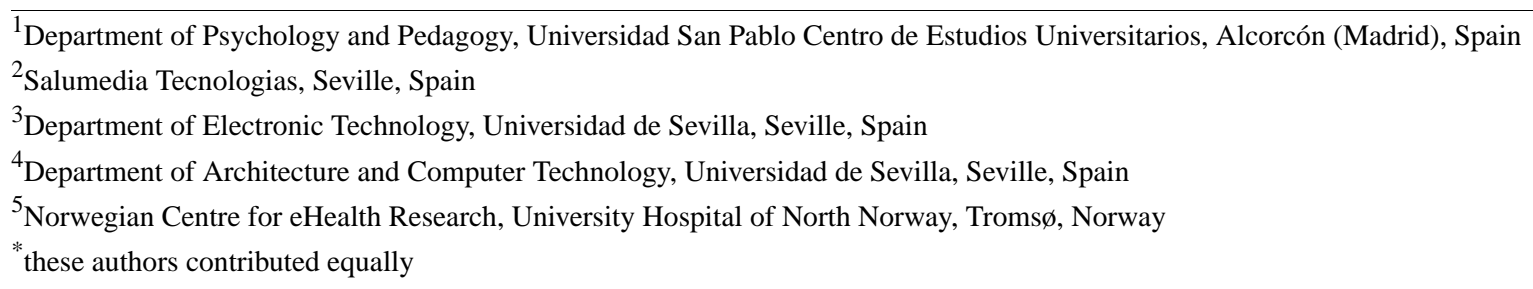

Corresponding Author:

Esther Rincon, PhD

Department of Psychology and Pedagogy

Universidad San Pablo Centro de Estudios Universitarios

Campus de Montepríncipe, Urbanización Montepríncipe, Pabellón MED, Despacho 0022

Alcorcón (Madrid), 28925

Spain

Phone: 34913724700 ext 4609

Fax: 34913724700

Email: maria.rinconfernande@ceu.es

\section{Abstract}

Background: Mobile phone health apps are increasingly gaining attention in oncological care as potential tools for supporting cancer patients. Although the number of publications and health apps focusing on cancer is increasing, there are still few specifically designed for the most prevalent cancers diagnosed: breast and prostate cancers. There is a need to review the effect of these apps on breast and prostate cancer patients' quality of life (QoL) and well-being.

Objective: The purposes of this study were to review the scientific literature on mobile phone apps targeting breast or prostate cancer patients and involving QoL and well-being (anxiety and depression symptoms) and analyze the clinical and technological characteristics, strengths, and weaknesses of these apps, as well as patients' user experience with them.

Methods: We conducted a systematic review of peer-reviewed literature from The Cochrane Library, Excerpta Medica Database, PsycINFO, PubMed, Scopus, and MEDLINE to identify studies involving apps focused on breast and/or prostate cancer patients and QoL and/or well-being published between January 1, 2000, and July 12, 2017. Only trial studies which met the inclusion criteria were selected. The systematic review was completed with a critical analysis of the apps previously identified in the health literature research that were available from the official app stores.

Results: The systematic review of the literature yielded 3862 articles. After removal of duplicates, 3229 remained and were evaluated on the basis of title and abstract. Of these, 3211 were discarded as not meeting the inclusion criteria, and 18 records were selected for full text screening. Finally, 5 citations were included in this review, with a total of 644 patients, mean age 52.16 years. Four studies targeted breast cancer patients and 1 focused on prostate cancer patients. Four studies referred to apps that assessed QoL. Only 1 among the 5 analyzed apps was available from the official app store. In 3 studies, an app-related intervention was carried out, and 2 of them reported an improvement on QoL. The lengths of the app-related interventions varied from 4 to 12 weeks. Because 2 of the studies only tracked use of the app, no effect on QoL or well-being was found.

Conclusions: Despite the existence of hundreds of studies involving cancer-focused mobile phone apps, there is a lack of rigorous trials regarding the $\mathrm{QoL}$ and/or well-being assessment in breast and/or prostate cancer patients. A strong and collective effort should be made by all health care providers to determine those cancer-focused apps that effectively represent useful, accurate, and reliable tools for cancer patients' disease management.

Trial Registration: PROSPERO CRD42017073069; https://www.crd.york.ac.uk/PROSPERO/display_record.asp?ID= CRD42017073069 (Archived by WebCite at http://www.webcitation.org/6v38Clb9T) 
(JMIR Mhealth Uhealth 2017;5(12):e187) doi: 10.2196/mhealth.8741

\section{KEYWORDS}

cancer; mHealth; app; mobile phone; quality of life; well-being

\section{Introduction}

The number of new cancer cases diagnosed every year worldwide is rapidly rising from 14.1 million in 2012 to well over 20 million predicted by 2030 [1]. Of those, breast and prostate cancers are the most prevalent diagnosed in women and men, respectively [1]. It should be noted that around 30\% to $40 \%$ of these cancer patients suffer from psychological distress (anxiety and depression symptoms commonly reported) as has been mentioned previously by a meta-analysis comprising 94 studies and 14,078 cancer patients [2]. This emotional distress has been associated with poorer quality of life (QoL) [3]. Well-being, QoL, and treatment satisfaction in breast and prostate cancer patients could be monitored by ubiquitous technologies such as mobile phone health apps, which can provide useful data to reflect on therapy work [4] and thereby improve patients' well-being.

Mobile phone health apps have the potential to revolutionize psychological science because they can collect behavioral data [5] and behavioral information with great ecological validity [6], facilitating high-frequency assessments and more objective data collection [7]. These apps can also potentially empower patients, promoting behavior changes, facilitating self-monitoring of symptoms [8], improving their educational level [9], and allowing patients the feeling of being in contact with their health care team [10].

Apps are widely used by professionals and patients, and attention to them in health care environments is increasing daily [9]. However, there are some important concerns about their use. Because of the large number of health care apps available, patients could get overwhelmed, encountering difficulties in finding the right app or features [11]. Poorly validated information, often created by nonexperts [12], and a lack of updated data [13] have also been mentioned as concerning issues related to health apps. Limited evidence involving these apps in studies [14] and little or no quality control or regulations to guarantee the apps as user-friendly, accurate, or efficacious tools [15] have also been reported.

In several systematic reviews on mobile phone health apps, authors urge different strategies that will result in higher quality evidence for app effectiveness and contents [8,13,16-20]. This would allow us to distinguish apps that subscribe to evidence-based protocols from those that do not [21]. The health care team should have a leading role not only in the review and verification of app contents but also in determining the most reliable ones and in selecting the patients best suited to using them [13]. Therefore, health care providers and organizations should standardize the identification, evaluation, and selection of these mobile health (mHealth) apps to maximize their utility and safety [15].

Attending to patients' point of view about using mHealth apps, authors have commonly used survey studies to determine the user experience. In general, cancer patients positively value the use of Internet-based technologies for health care management and feel comfortable using them [22]. Breast cancer patients usually use this technology to seek general information, search for therapies or scientific data, and exchange information with other patients [23]. Authors have also pointed out the importance of including customizable functionalities in mobile phone apps in order to manage care-related information so that these features can be easily modified depending on changes in the user's needs [24]. Other people affected by cancer, such as prostate cancer patients, have shown interest in using apps, indicating apps should be easy to use, tailored to the individual, and include social support [25]. A recent survey of 375 cancer patients reported that about half of the patients $(182 / 375,48.5 \%)$ were willing to send data via an app supporting their oncological treatment and follow-up [4]. Moreover, around two-thirds $(125 / 182,68.7 \%)$ agreed to use these regularly sent data as an ideal complement to the standard follow-up. The most mentioned characteristics that should be included in a cancer-focused app were pseudonymizing, data protection, and feedback from a physician based on the patients' input [4].

Although mHealth apps could be useful tools for cancer patients [26], there are only a few apps focused on oncological care that support patients during treatment and aftercare [4]. The purposes of this study were to (1) identify evidence-based mobile phone health apps focused on QoL and well-being (anxiety and depression symptoms) in breast and/or prostate cancer patients, (2) recognize their clinical and technological characteristics, (3) categorize their clinical and technological strengths and weaknesses, and (4) determine patients' user experience (satisfaction level and comments regarding the apps used).

\section{Methods}

\section{Overview}

We developed a systematic search strategy to detect all relevant studies involving the use of mobile phone apps for QoL and/or well-being (anxiety and depression symptoms) in breast and/or prostate cancer on July 12, 2017. Once we determined these studies, we searched the identified apps on the online market to describe them. The systematic research protocol is registered at PROSPERO [CRD42017073069].

\section{Reviewing the Scientific Literature}

\section{Selection Criteria}

Articles were considered potentially relevant if they were trials or peer-reviewed studies published between January 1, 2000, and July 12, 2017, including a smartphone app focused on QoL and/or well-being (anxiety and depression symptoms) used by breast and/or prostate cancer patients.

We considered a smartphone "a mobile phone with Internet connectivity and the ability to download and run third-party software apps available from a commercial marketplace" [27]. 
We excluded articles that did not involve a mobile phone app (eg, a Web-based or iPad app), medical studies, systematic reviews and meta-analyses, abstracts or congress papers, qualitative studies, study protocols, and studies not including QoL or well-being assessment. We applied no language restrictions.

The search strategy followed the Preferred Reporting Items for Systematic Reviews and Meta-Analysis (PRISMA) guidelines [28]. We searched for trials in The Cochrane Library, Excerpta Medica Database (EMBASE), PsycINFO (via ProQuest), PubMed, Scopus, and MEDLINE (via OvidSP) on July 12, 2017. We extracted trials with the keywords "breast cancer + app," "breast cancer + mHealth," "breast cancer + mobile application," "prostate cancer + app," "prostate cancer + mHealth," and "prostate cancer + mobile application" published between January 1, 2000, and July 12, 2017. Two of the authors of this study (ER and EG) independently reviewed the titles and abstracts of the total search yield to identify eligible articles. The full text of the article was retrieved if any reviewer considered a citation potentially relevant. In case of disagreement, a third reviewer (FG) selected the reference finally included, based on inclusion and exclusion criteria. Search results were stored using Endnote version X8 (Clarivate Analytics). Duplications of studies were removed.

\section{Data Extraction}

Two of the authors of this study (ER and EG) independently reviewed the full text of the articles meeting the eligibility criteria. The interrater agreement (kappa value) was calculated with SPSS version 22 (IBM Corp). The following data were extracted from the selected papers: (1) general patient and study characteristics (year of publication, country of study, language, author affiliations, number of participants, mean age, and cancer type targeted), (2) clinical characteristics (QoL assessment, other variables measured, functionalities, type of validated questionnaire involved and timing for assessment, treatment offered, main clinical results, quality of the study, randomized controlled trial [RCT] design, social media inclusion, theoretical framework based, and quality of the journal), (3) clinical strengths and weaknesses, and (4) patients' user experience (satisfaction level and comments regarding the apps used). Disagreements were rare and were easily resolved by consensus.

Two of the authors (ORR and ED) independently reviewed the full text of selected articles and extracted the following information: (1) technological characteristics (app name, platform, availability in markets, price, number of downloads, rating, patients targeted, and main features), (2) technological strengths and weaknesses, and (3) patients' user experience (ratings, health certification obtained, and number of user comments). Disagreements were resolved by a third reviewer (FG). The quality of the included studies was assessed in terms of their design. Nonrandomized, observational, descriptive, and qualitative studies were considered low to medium quality. Quasi-randomized and interventional studies and studies with strongest design were considered of moderate to high quality.

\section{Reviewing the Apps on the Market}

In addition to the systematic review, 3 of the authors (ORR, $\mathrm{ED}$, and CSB) downloaded the apps identified in the studies from the online store. They collected the following information: (1) technological characteristics, (2) technological strengths and weaknesses, and (3) patients' user experience (satisfaction level and comments regarding the app used). An English language restriction was applied for the mobile phone apps downloaded.

\section{Results}

\section{General Characteristics}

The search of the electronic databases retrieved 3862 citations. After removal of duplicates, 3229 remained and were evaluated on the basis of title and abstract. Of these, 3211 were discarded because they clearly did not meet the inclusion criteria. Based on titles and abstracts, 18 records were selected for full text screening; 13 out of these 18 [29-41] being discarded for various reasons (see Multimedia Appendix 1). A total of 5 publications [42-46] were finally included. An interrater agreement of kappa=.561 was found, reaching a moderate agreement according to Landis and Koch [47]. All chosen studies were deemed to be of sufficient quality to contribute equally to the thematic synthesis. A PRISMA flowchart is shown in Figure 1 [28].

The 5 studies included a total of 644 patients, mean age 52.16 years (sample sizes and mean ages listed in Table 1). Of these, 3 studies were conducted in Korea [42,44,46], 1 in the United States [43], and 1 in Sweden [45]. All main authors affiliations were university departments [42-46]. The majority of the studies targeted breast cancer patients [42-44,46]; only 1 focused on prostate cancer patients [45]. Other general characteristics of the studies included are summarized in Table 1.

\section{Clinical and Technological Characteristics}

Regarding the clinical approach, 4 of the 5 included studies referred to apps that assessed QoL [43-46]. Among the other variables measured were depression status (mood, anxiety, and sleep satisfaction) [42]; daily food intake, daily exercise, daily body weight, weight efficacy, anthropometrics, and physical activity [43]; sleep disturbance [44]; sense of coherence, bladder and bowel function, fatigue, pain, anxiety, distress, sleep, and flushing [45]; physical activity [43,46]; and user satisfaction [46].

All studies allowed patients to collect patient-reported outcome measures [42-46], and 3 included a related-intervention app $[43,45,46]$. 
Figure 1. Systematic review of the literature flowchart.
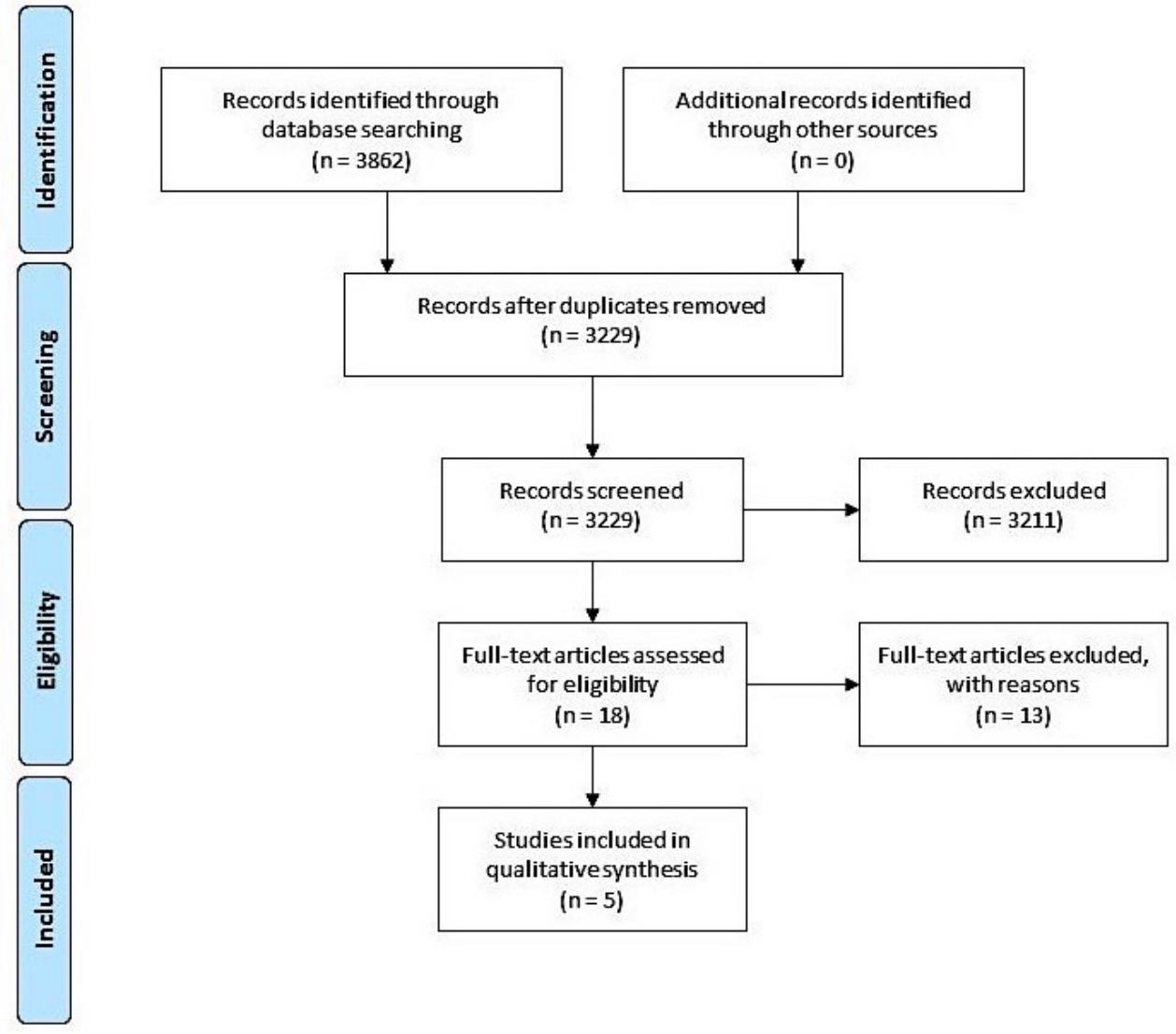

Table 1. General characteristics of included studies $(n=5)$.

\begin{tabular}{|c|c|c|c|c|c|}
\hline Study & Publication year & Country/language & Participant number & Mean age & Cancer type \\
\hline Kim et al [42] & 2016 & Korea/Korean & 78 & 44.35 & Breast \\
\hline McCarroll et al [43] & 2015 & United States/English & 50 & 58.4 & Breast \\
\hline Min et al [44] & 2014 & Korea/Korean & 30 & 45 & Breast \\
\hline Sundberg et al [45] & 2017 & Sweden/Swedish & 130 & 69 & Prostate \\
\hline Uhm et al [46] & 2017 & Korea/Korean & 356 & 50.3 & Breast \\
\hline
\end{tabular}

The included studies measured QoL through different questionnaires such as the Functional Assessment of Cancer Therapy-General (FACT-G) [48], a generic core questionnaire that comprises 27 items divided into 4 domains (physical, functional, emotional, and social well-being) [49]; the EuroQol 5 Dimensional Questionnaire (EQ-5D-3L) [50], a generic health outcome instrument comprising 5 dimensions (mobility, self-care, usual activities, pain/discomfort, and anxiety/depression) [49]; the European Organization for Research and Treatment of Cancer Quality of Life Questionnaire-Core (EORTC QLQ-C30) [51], a 30-item generic cancer questionnaire that consists of 5 function scales (physical, role, emotional, cognitive, and social), a global health scale, 3 multi-item symptom scales (fatigue, nausea/vomiting, and pain), and 6 single item scales (dyspnea, sleep, appetite, constipation, diarrhea, and financial difficulties due to disease) [49]; and the EORTC complementary modules on prostate cancer, QLQ-PR25 [52], and breast cancer, QLQ-BR23 [53]. One study did not assess QoL symptoms [42], focusing only on well-being assessment through the Patient Health Questionnaire (PHQ-9)
[54], which measures both the presence and the severity of 9 depression symptoms and is able to yield a diagnosis [55]. Only one of the included studies reported the theoretical framework on which the app relied. Further details on other clinical variables assessed by the included studies are reported in Table 2 .

Concerning the main clinical results, the adherence to the self-reporting measures was associated with higher accuracy of depression screening [42]. Moreover, the compliance with the daily self-reporting rates was not affected by depression symptoms or health-related quality of life (HRQoL) status reported by the patients [44]. Of the 3 studies that included intervention $[43,45,46]$, only 2 reported a QoL improvement $[45,46]$. The real-time assessment and management of symptoms through Interaktor [45] produced significantly less burden in emotional functioning, insomnia, and urinary-related symptoms at T2 (after end of treatment, which ranged from 5 to 8 weeks) and at T3 (3 months after end of treatment) compared with the control group. Likewise, the 12-week regimen of aerobic and 
resistance exercise through Smart After Care [46] produced a significant improvement in physical function, physical activity, and QoL at baseline and 12 weeks. The 4-week comprehensive lifestyle program focusing on nutrition quality, physical activity, and improving eating displayed by LoseIt! [43] did not affect QoL.

With regard to the quality of the studies, 3 were considered of low-to-medium level $[42,43,44]$, and 2 a medium-to-high level of quality $[45,46]$.

The study design involved 2 prospective, nonrandomized multicenter controlled trials [45,46], 1 with control group [45], but no RCT designs were included. None of the 5 studies involved social media features, and only 1 study [43] mentioned the theoretical framework, social cognitive theory, on which it was based.

Concerning the publication journals, 4 out of the 5 included studies $[42,43,45,46]$ were published in the last 3 years (2015-2017). Scientific journals are ranked yearly based on impact factor data, and the Journal Citation Reports (JCR) published by Clarivate Analytics are widely used as a quality indicator. The JCR ranks journals into categories based on which quartile of the impact factor distribution the journal occupies for that category: Q1 represents the top 25\% of journals in the distribution, Q2 between the top 50\% and top 25\% of journals, and Q3 between the top $75 \%$ and top $50 \%$ of journals. According to the $2017 \mathrm{JCR}, 4$ studies [42-44,46] were issued in journals ranking Q1 or Q2.

With regard to technological characteristics, all of the 5 studies included provided the names of the mobile phone apps evaluated [42-46]. There were 2 studies involving the same app [42,44] (see Table 3). The majority of the studies included an app targeted at cancer patients [42,44-46]. The main features of the apps were focused on exercise and nutrition logging [43]; collection of patient-reported outcomes [44]; early detection, reporting, and management of symptoms [45]; and exercise by a step counter [46]. More technological characteristics can be seen in Table 3.

App functionalities included the following: (1) customization and personalization features (create a menu plan and calorie tracker [43] to establish an individual profile), (2) motivational features (providing feedback), and (3) social features (developing a community for inspiration).

Table 2. Clinical characteristics of included studies $(n=5)$.

\begin{tabular}{|c|c|c|c|c|c|}
\hline Study & $\begin{array}{l}\text { QoL }^{\mathrm{a}} \\
\text { assessment }\end{array}$ & Functionalities & Validated questionnaire/timing & $\begin{array}{l}\text { Treatment } \\
\text { offered }\end{array}$ & Quality of study \\
\hline Kim et al [42] & No & $\begin{array}{l}\text { PRO }^{\text {b }} \text { : daily mental health ratings } \\
\text { over a } 48 \text {-week period }\end{array}$ & PHQ-9 ${ }^{\mathrm{c}}$ via app biweekly & No & Low-medium \\
\hline McCarroll et al [43] & Yes & $\begin{array}{l}\text { PRO: daily, real-time, and motiva- } \\
\text { tional feedback + intervention }\end{array}$ & $\begin{array}{l}\text { FACT-G }^{\mathrm{d}}, \mathrm{WEL}^{\mathrm{e}} \text { at baseline and at } \\
\text { 4-week follow-up }\end{array}$ & $\begin{array}{l}\text { Comprehensive } \\
\text { lifestyle pro- } \\
\text { gram }\end{array}$ & Low-medium \\
\hline Min et al [44] & Yes & $\begin{array}{l}\text { PRO: daily basis over a } 90 \text {-day peri- } \\
\text { od }\end{array}$ & $\begin{array}{l}\mathrm{BDI}^{\mathrm{f}}, \mathrm{EQ}-5 \mathrm{D}-3 \mathrm{~L}^{\mathrm{g}} \text { via app on a daily } \\
\text { basis for } 90 \text { days }\end{array}$ & No & Low-medium \\
\hline Sundberg et al [45] & Yes & $\begin{array}{l}\text { PRO: daily, real-time assessment of } \\
\text { symptoms and concerns during ra- } \\
\text { diotherapy + intervention }\end{array}$ & $\begin{array}{l}\text { EORTC QLQ-C } 300^{\mathrm{i}} \text {, EORTC QLQ- } \\
\text { PR } 25^{\mathrm{j}} \text { via app daily at any time } \\
\text { during radiotherapy and } 3 \text { weeks } \\
\text { after completion }\end{array}$ & $\begin{array}{l}\text { Management of } \\
\text { symptoms }\end{array}$ & Medium-high \\
\hline Uhm et al [46] & Yes & PRO + intervention ${ }^{\mathrm{h}}$ & $\begin{array}{l}\text { EORTC QLQ-C30, EORTC QLQ- } \\
\text { BR23k at baseline and } 12 \text { weeks }\end{array}$ & $\begin{array}{l}\text { 12-week regi- } \\
\text { men of aerobics }\end{array}$ & Medium-high \\
\hline
\end{tabular}

\footnotetext{
${ }^{\mathrm{a}} \mathrm{QoL}$ : quality of life.

${ }^{\mathrm{b}} \mathrm{PRO}$ : patient-reported outcome measures.

${ }^{\mathrm{c}}$ PHQ-9: Patient Health Questionnaire-9.

${ }^{\mathrm{d}}$ FACT-G: Functional Assessment of Cancer Therapy-General.

${ }^{\mathrm{e}}$ WEL: Weight Efficacy Lifestyle questionnaire.

${ }^{\mathrm{f}} \mathrm{BDI}$ : Beck Depression Inventory.

${ }^{\mathrm{g}}$ EQ-5D-3L: EuroQol 5-Dimension 3-Level survey.

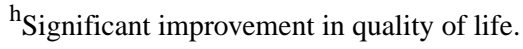

${ }^{\mathrm{i}}$ EORTC QLQ-C30: European Organization for Research and Treatment of Cancer Quality of Life Questionnaire-Core.

${ }^{j_{E}}$ EORTC QLQ-PR25: European Organization for Research and Treatment of Cancer Quality of Life Questionnaire-Prostate.

kEORTC QLQ-BR23: European Organization for Research and Treatment of Cancer Quality of Life Questionnaire-Breast Cancer.
} 
Table 3. Technological characteristics of included studies $(n=5)$.

\begin{tabular}{|c|c|c|c|c|c|c|c|}
\hline Study & App name & Platform & $\begin{array}{l}\text { Available in } \\
\text { markets }\end{array}$ & Price & Downloads & Ratings & $\begin{array}{l}\text { Patients } \\
\text { Targeted }\end{array}$ \\
\hline Kim et al [42] & Pit-a-Pat & Android/iOS & No & Unknown & Unknown & Unknown & Yes \\
\hline McCarroll et al [43] & LoseIt! & Android/iOS & Yes & free/premium & $\begin{array}{l}\text { Android: } \\
5,000,000- \\
10,000,000\end{array}$ & $\begin{array}{l}\text { Android: } 4.4 ; \\
\text { iOS: } 4.0\end{array}$ & No \\
\hline Sundberg et al [45] & Interaktor & Unknown & No & Unknown & Unknown & Unknown & Yes \\
\hline Uhm et al [46] & Smart After Care & iOS & No & Unknown & Unknown & Unknown & Yes \\
\hline
\end{tabular}

\section{Clinical and Technological Strengths and Weaknesses}

Regarding the studies' strengths, 2 of them involved the same app called Pit-a-Pat [42,44]. Pit-a-Pat was developed for cancer patients to self-report factors related with the diagnosis itself and the subsequent treatments: (1) sleep-disturbance symptoms, (2) acute symptoms related to cytotoxic chemotherapeutic agents, and (3) medication diary for antihormonal treatment. Kim et al [42] studied the accuracy of a mobile mental health tracker for depression screening, as well as the adherence on screening accuracy. For that purpose, daily patient reports of anxiety symptoms, mood, and sleep satisfaction were collected through a reliable and valid questionnaire (PHQ-9). The app involved user-friendly functionalities in the form of a facial emoticon scale. Min et al [44] studied the patient's self-reported sleep disturbance, HRQoL status, and depression symptoms via the Pit-a-Pat app on a daily basis for 90 days with standardized questionnaires. Push notifications were sent to participants daily at $9 \mathrm{AM}$ and $7 \mathrm{PM}$.

LoseIt! [43] was a Web- and mobile-based app, not cancer targeted, used for logging food intake and volitional exercise. McCarroll et al [43] aimed to assess a 4-week comprehensive lifestyle program with emphasis on nutrition quality, physical activity, and improving eating self-efficacy delivered using a beta health care provider version of LoseIt! in which the patients could log daily food choices, daily exercise type and duration, and daily body weight over the treatment period. Participants received motivational patient-provider feedback notifications (phone call, email message, and/or a push notification) in response to their individual input in the LoseIt! app.

Interaktor [45] was codesigned by patients and health care personnel as an interactive app for mobile phones and tablets. Interaktor was specifically intended for early detection, reporting, and management of symptoms and concerns during treatment for prostate cancer. Daily reports via the app enable instant support from a nurse in early detection and management of symptoms and concerns in real-time during treatment for prostate cancer. The app features included symptom assessment, a risk assessment model for alerts directly to a nurse, continuous access to evidence-based self-care advice, and links to relevant websites. The apps sent patients a reminder message if they had not submitted their report. In addition, the system sent 2 alerts (yellow and red) to the patients, depending on their symptom occurrence and frequency. The red meant a higher priority (should contact the nurse within an hour), and the yellow alert indicated that the nurse should be called that day. Sundberg et al [45] included a control and an intervention group in their study, which used the app for daily, real-time assessment and management of symptoms and concerns during radiotherapy treatment. Participants were asked to send reports daily and at any time point when they felt unwell during the radiation treatment ( 5 to 8 weeks) and the following 3 weeks.

Smart After Care [46] was a newly developed mobile phone exercise app. This app recorded minutes of physical activity weekly and established a weekly goal for minutes of activity beginning in the second week. Every week, the achievement rate was displayed by the app. Also, patients receiving hormonal therapy could watch a video clip of resistance and stretching exercises through the app. This study included standardized QoL questionnaires and a user satisfaction survey in the intervention group. Patients in the study by Uhm et al [46] performed a 12-week regimen of aerobic and resistance exercise through Smart After Care, where the intervention group received a pedometer and Smart After Care to perform 150 or 90 minutes of aerobic exercise.

Among the main weaknesses could be cited the small samples of the studies [42-44] and the lack of RCT protocols and framework-based apps. Moreover, we can report only 1 app for free download on the market [43], which was used with breast cancer patients despite not being cancer-focused.

\section{Patients' User Experience}

Only 1 study [46] reported information regarding patient satisfaction level, and only 1 app showed a quality certification [43] and a considerable number of user comments (see Table 4). The mean Likert scale response for overall patient satisfaction with the service was $4.27 / 5$ in the mHealth group [46]. 
Table 4. Patient satisfaction levels provided by the health literature review and the online store search $(n=5)$.

\begin{tabular}{lllll}
\hline Study & Patients satisfaction & Ratings $\left(\mathrm{OSR}^{\mathrm{a}}\right)$ & Health certification (OSR) & Number of user comments (OSR) \\
\hline Kim et al [42] & Unknown & Unknown & Unknown & Unknown \\
McCarroll et al [43] & Unknown & Android: 4.4; iOS: 4.0 & $\begin{array}{l}\text { Helix's CLIA }{ }^{\mathrm{b}} \text { certified and CAP } \\
\text { accredited lab }\end{array}$ & Android: 61,063; iOS: 374,815 \\
Min et al [44] & Unknown & Unknown & Unknown & Unknown \\
Sundberg et al [45] & Unknown & Unknown & Unknown & Unknown \\
Uhm et al [46] & Satisfied with use & Unknown & Unknown & Unknown \\
\hline
\end{tabular}

${ }^{\mathrm{a} O S R}$ : Online store research.

${ }^{\mathrm{b}}$ CLIA: Certified Laboratory Improvement Amendments.

${ }^{\mathrm{c}} \mathrm{CAP}$ : College of American Pathologists.

\section{Discussion}

\section{Overview}

The use of mobile phone apps for health purposes continues to increase [7], and currently thousands and thousands of health apps are available on the online market. They target different health conditions, including cancer. Health apps represent an opportunity to monitor psychological distress and QoL related to cancer and its associated treatments. Our systematic review shows that the scientific literature referring to apps targeting breast or prostate cancer patients and involving QoL and/or well-being measurements is very modest, as we only could identify 5 studies meeting the inclusion criteria. However, the quality and dates of publication show a current scientific interest in this research topic.

The most recent reviews involving focused cancer apps started the searching methodology by looking for apps on the online stores, followed by searching bibliographic databases of health literature $[8,13]$. However, we considered it more appropriate to start by determining whether rigorous trials had been published on cancer-focused apps. Hence, we conducted the systematic literature review first, and then we downloaded the apps from the market stores to examine them.

\section{More Evidence-Based Apps Are Needed}

Despite the increase in the number of health care apps available [15], only a very few of them discussed in the scientific literature focus on QoL and/or well-being assessment in breast or prostate cancer patient even though breast and prostate cancer are the most prevalent cancers diagnosed [1] and QoL and well-being are frequently assessed to determine the health status of cancer patients [2,3]. There are only 2 studies that reported QoL improvement by using related-treatment health apps $[45,46]$.

Related research on health apps for cancer patients was identified but not included in the review due to the following reasons (see Multimedia Appendix 1): it did not involve mobile phone apps [30-32,35,37,38], it did not assess QoL or well-being $[29,33,34,36,40]$, or it involved qualitative studies focused on feasibility or patient opinions $[39,41]$.

We selected the QoL measure considering it has a wide range of variables involving other psychological measures (eg, cognitive, emotional, and social abilities) and not only as performance status and daily functional activities [29], symptom experience [33], or chemotherapy-related symptoms (nausea, vomiting, fatigue, mucositis, hand-foot syndrome, and diarrhea) [34].

We have defined well-being as existing levels of general anxiety and depression symptoms and not only as perceived stress level [36] assessed by the Perceived Stress Scale (PSS) [56], a scale "designed to measure the degree to which situations in one's life are appraised as stressful" and suggested by its own authors "as an outcome measure of experienced levels of stress" [56], not well-being levels.

In our review, only 1 of the selected papers provided information regarding patient satisfaction level [46], therefore it is not possible to draw conclusions about the patient satisfaction or perceived effectiveness of the current apps.

Most of the apps referred to in the scientific literature targeted breast cancer, as in previous reviews [8,13,16-18], and only 1 study focused on prostate cancer. All of these health apps were developed by university institutions; in contrast with the review of Mobasheri et al [18], which reports that a minority of medical professionals were involved in the apps. Our results showed that, a priori, all the studies have been hosted by significant research and educational institutions.

With regard to the technological characteristics, it should be noted that the 3 apps specifically designed for cancer patients [42,44-46] were not available for download on the market. Furthermore, only 1 [43] out of 4 total apps included was available for download at the online store, and despite this app (LoseIt!) not being specifically patient-targeted, it was used by 50 breast cancer patients to manage exercise and nutrition concerns.

\section{Clinical and Technological Strengths and Weaknesses}

Regarding the studies' strengths, the use of related-treatment mobile phone apps has resulted in a significant improvement in cancer patients' QoL $[45,46]$. Some features like displaying daily patient reports in real time and providing personalized feedback $[43,45]$ have also been pointed out as a significant advantage of the apps [34]. Moreover, if the assessment involves user-friendly functionalities such as a facial emoticon scale [42], which could be adapted to the small phone screen, this may facilitate user participation, potentially making the data more 
useful. Previous studies [31] have reported important usability adaptation, incorporating several design decisions to account for patients with various disabilities (eg, impaired vision), presenting only 1 question at a time to patients. The easy and visual (similar to a stock chart) way of obtaining the information displayed by the app as feedback on the symptoms report form at any time during the study has also been mentioned as a relevant strength [29]. Also, it is notable that when some researchers wished to test a general-population-targeted app with cancer patients, they delivered a cancer-focused health care provider beta version [43] and designed the user interface of the app based on reliable guidelines developed by the National Cancer Institute, like previous authors did [31].

Participants using their own mobile phones have mentioned this as better than being provided with an additional device [44], probably because in the latter case they must deal with 2 mobile phones in their daily life or because they prefer some relative freedom for testing the app, meaning the possibility of using the app at their convenience with no minimum amount of time to be spent using the app, as previous authors have reported [41]. In contrast, other studies have pointed out patient preferences for using a device without phone functionality [57] instead of using their own phones. Users strongly appreciate the use of no personal patient identifiers or other information stored on the devices used [30], as well as pseudonymizing and data protection [4]. Apps that can be used on more than 1 device could provide the patients with more possibilities to test them, such as LoseIt! [43], which offers both website and mobile versions for users, or Interaktor [45], available for mobile phones and tablets. The real-time feedback component and the motivational feedback notification are considered relevant strengths as well. The flexibility in the self-reporting task [45] could probably be a more suitable option in oncological settings than prefixed hours of a day [44] because of the patients' highly variable functional status during the day, largely dependent on the medical treatments.

Among the main weaknesses could be (1) no cancer-focused apps are being used in studies involving cancer patients [36,40,41,43,57], (2) many of these studies have small samples, (3) studies are without rigorous design based on RCTs, (4) studies are not free to the user, (5) no theoretical framework is reported, and (6) there are usability and accessibility issues with cancer patients.

It is important to highlight the relevance of using cancer-focused apps in oncological settings, as cancer patients could be considered vulnerable recipients [4]. People suffering from cancer have to struggle with quick relapses, bad prognoses [4], side effects caused by cancer treatments, and psychological distress [2]. Also, they represent a population interested in doing everything possible to improve their health [4], so they could be interested in using apps that do not constitute reliable and accurate tools for them, which is even worse if the patients have to pay for them. Mobasheri et al [18] reported that of the 30 apps reviewed, which functioned as self-assessment tools for breast disease, only $2(2 / 30,7 \%)$ had a documented evidence base (the rest relied on empirical data). It is imperative to develop apps and other health information and technology systems specifically targeted to cancer patients.
Although some encouraging results have been reported using apps in cancer patients [34], bigger samples sizes and framework-based and RCT designs are needed in order to obtain stronger research conclusions. Otherwise, serious concerns could arise regarding the lack of validation [12] and quality control [15] of the studies. Furthermore, the identification of rigorous trials involving empirical testing of these mobile phone apps in oncological settings is imperative, as none of the selected studies in this review followed a randomized method and only 1 was based on a previous framework. The use of theories, models, and frameworks for apps will help to identify the mechanisms, approaches, and functionalities that work best.

Cancer patients and survivors could have cognitive deterioration due to treatments. Therefore, usability and accessibility are relevant aspects to be considered in the development process of these apps, especially when they are intended for older people. Apps not designed for cancer patients and survivors could entail difficult challenges for them, resulting in reduced adoption and engagement rates. Equally, the large variety of apps available makes it difficult to establish which of them are the most adequate for breast and prostate cancer patients and what is the best way to use them. Also, patients could become overwhelmed due to the huge number of cancer apps available [11]. Because of this, stronger efforts should be made to consolidate the evidence base, effectiveness, and safety of cancer-focused apps [8].

In line with previous research [8,13,16-18,20,21], we consider a main challenge the task of ensuring that those apps that are planned to be used with cancer patients be effectively cancer-focused, meaning that they should have been originally designed for, tested on, and adapted to the cancer population. Only if this technology is evidence-based and targeted to cancer patients can health care providers guarantee the apps' safety, accuracy, reliability, and high quality and be able to recommend them in oncological care settings.

\section{Patients' Satisfaction With the Health Apps}

Regarding patients' satisfaction, it is noteworthy that only 1 app of the 3 reviewed reported a quality certification and showed user comments regarding its use. Moreover, it is relevant that this app was not cancer-focused and was the only one available for download at the online store. Only user-friendly and quality-certified apps should be provided to cancer patients. Thus, these health apps must be available for download at market stores once they are certified as useful tools for cancer patients. It would probably be helpful as well that these apps provide new users with comments about other patient's experiences, in order to obtain a more powerful overview of the main features included in the app.

More evidence on the patient satisfaction level using health apps for QoL and/or well-being assessment in oncological settings is needed. In our review, only 1 study [46] focusing on cancer patients provided information about satisfaction level.

People affected by cancer are usually open to strategies that could have a positive influence on their disease [4]. Probably due to this fact, mobile phone developers and health care teams involved in oncological settings should be especially careful 
with the apps that are going to be used and tested by this population and implement patient-centered design approaches. Current RCTs are still being developed that might produce promising data to help reach a high-quality evidence base for apps for cancer patients' use [32,57-59].

\section{Limitations}

Our study had certain limitations. Our selection criteria intentionally excluded apps that were not specifically focused on breast or prostate cancer patients. We considered only the assessment of 2 main psychological variables in psycho-oncological care: QoL and well-being (anxiety and depression symptoms). Additional studies could consider other psychological measures such as fatigue or the secondary symptoms produced by the cancer treatments. Although our data search represents a wide range of peer-reviewed journals, we might have missed studies that were not identified with our search terms or that were not published.

\section{Conclusions}

Despite the existence of hundreds of studies involving mobile phone health apps used by cancer patients, there is a lack of rigorous trials regarding QoL and/or well-being assessment in breast and/or prostate cancer patients. More evidence-based apps, which could be tested in future RCT protocols, are still needed. However, promising results are expected to be available from some RCTs that are still running. A strong and collective effort should be made by all health care providers to determine those cancer-focused apps that provide useful and reliable tools for cancer patients' disease management.

\section{Acknowledgments}

This study received funding from the European Union's Horizon 2020 research and innovation program under the Marie Sklodowska-Curie grant agreement number 722012 (Cancer: Activating Technology for Connected Health). The authors would like to sincerely thank Dr Luis Fernandez-Luque for his guidance and support.

\section{Conflicts of Interest}

None declared.

\section{Multimedia Appendix 1}

Reasons for exclusion of studies.

[PDF File (Adobe PDF File), 26KB-Multimedia Appendix 1]

\section{Multimedia Appendix 2}

Overview of the systematic review.

[PDF File (Adobe PDF File), 11MB-Multimedia Appendix 2]

\section{References}

1. Bray F. Transitions in human development and the global cancer burden. In: Stewart BW, Wild CP, editors. World Cancer Report 2014. Lyon: International Agency for Research on Cancer; 2014:54-68.

2. Mitchell AJ, Chan M, Bhatti H, Halton M, Grassi L, Johansen C, et al. Prevalence of depression, anxiety, and adjustment disorder in oncological, haematological, and palliative-care settings: a meta-analysis of 94 interview-based studies. The Lancet Oncology 2011 Feb;12(2):160-174. [doi: 10.1016/S1470-2045(11)70002-X] [Medline: 21251875]

3. Giese-Davis J, Collie K, Rancourt KMS, Neri E, Kraemer HC, Spiegel D. Decrease in depression symptoms is associated with longer survival in patients with metastatic breast cancer: a secondary analysis. J Clin Oncol 2011 Feb 01;29(4):413-420 [FREE Full text] [doi: 10.1200/JCO.2010.28.4455] [Medline: 21149651]

4. Kessel KA, Vogel MM, Kessel C, Bier H, Biedermann T, Friess H, et al. Mobile health in oncology: a patient survey about app-assisted cancer care. JMIR Mhealth Uhealth 2017 Jun 14;5(6):e81 [FREE Full text] [doi: 10.2196/mhealth.7689] [Medline: 28615159]

5. Gosling SD, Mason W. Internet research in psychology. Annu Rev Psychol 2015 Jan 03;66:877-902. [doi: 10.1146/annurev-psych-010814-015321] [Medline: 25251483]

6. Harari GM, Lane ND, Wang R, Crosier BS, Campbell AT, Gosling SD. Using smartphones to collect behavioral data in psychological science: opportunities, practical considerations, and challenges. Perspect Psychol Sci 2016 Nov;11(6):838-854 [FREE Full text] [doi: 10.1177/1745691616650285] [Medline: 27899727]

7. Dorsey ER, Yvonne CY, McConnell MV, Shaw SY, Trister AD, Friend SH. The use of smartphones for health research. Acad Med 2017 Dec;92(2):157-160. [doi: 10.1097/ACM.0000000000001205] [Medline: 27119325]

8. Bender JL, Yue RYK, To MJ, Deacken L, Jadad AR. A lot of action, but not in the right direction: systematic review and content analysis of smartphone applications for the prevention, detection, and management of cancer. J Med Internet Res 2013 Dec 23;15(12):e287 [FREE Full text] [doi: 10.2196/jmir.2661] [Medline: 24366061] 
9. Mosa ASM, Yoo I, Sheets L. A systematic review of healthcare applications for smartphones. BMC Med Inform Decis Mak 2012 Jul 10;12:67 [FREE Full text] [doi: 10.1186/1472-6947-12-67] [Medline: 22781312]

10. Wang J, Wang Y, Wei C, Yao NA, Yuan A, Shan Y, et al. Smartphone interventions for long-term health management of chronic diseases: an integrative review. Telemed J E Health 2014 Jun;20(6):570-583. [doi: 10.1089/tmj.2013.0243] [Medline: 24787747]

11. van VL, Beaujean DJMA, van GPJEWC. Why mobile health app overload drives us crazy, and how to restore the sanity. BMC Med Inform Decis Mak 2013 Feb 11;13:23 [FREE Full text] [doi: 10.1186/1472-6947-13-23] [Medline: 23399513]

12. O'Neill S, Brady RRW. Colorectal smartphone apps: opportunities and risks. Colorectal Dis 2012 Sep;14(9):e530-e534. [doi: 10.1111/j.1463-1318.2012.03088.x] [Medline: 22646729]

13. Collado-Borrell R, Escudero-Vilaplana V, Ribed-Sánchez A, Ibáñez-García S, Herranz-Alonso A, Sanjurjo-Sáez M. Smartphone applications for cancer patients; what we know about them? Farm Hosp 2016 Jan 01;40(1):25-35 [FREE Full text] [doi: 10.7399/fh.2016.40.1.8993] [Medline: 26882831]

14. Mirkovic J, Kaufman DR, Ruland CM. Supporting cancer patients in illness management: usability evaluation of a mobile app. JMIR Mhealth Uhealth 2014 Aug 13;2(3):e33 [FREE Full text] [doi: 10.2196/mhealth.3359] [Medline: 25119490]

15. Boudreaux ED, Waring ME, Hayes RB, Sadasivam RS, Mullen S, Pagoto S. Evaluating and selecting mobile health apps: strategies for healthcare providers and healthcare organizations. Transl Behav Med 2014 Dec;4(4):363-371 [FREE Full text] [doi: 10.1007/s13142-014-0293-9] [Medline: 25584085]

16. Agboola SO, Ju W, Elfiky A, Kvedar JC, Jethwani K. The effect of technology-based interventions on pain, depression, and quality of life in patients with cancer: a systematic review of randomized controlled trials. J Med Internet Res 2015 Mar 13;17(3):e65 [FREE Full text] [doi: 10.2196/jmir.4009] [Medline: 25793945]

17. Brouard B, Bardo P, Bonnet C, Mounier N, Vignot M, Vignot S. Mobile applications in oncology: is it possible for patients and healthcare professionals to easily identify relevant tools? Ann Med 2016 Nov;48(7):509-515. [doi:

10.1080/07853890.2016.1195010] [Medline: 27348761]

18. Mobasheri MH, Johnston M, King D, Leff D, Thiruchelvam P, Darzi A. Smartphone breast applications-what's the evidence? Breast 2014 Oct;23(5):683-689. [doi: 10.1016/j.breast.2014.07.006] [Medline: 25153432]

19. Pandey A, Hasan S, Dubey D, Sarangi S. Smartphone apps as a source of cancer information: changing trends in health information-seeking behavior. J Cancer Educ 2013 Mar;28(1):138-142. [doi: 10.1007/s13187-012-0446-9] [Medline: 23275239]

20. Payne HE, Lister C, West JH, Bernhardt JM. Behavioral functionality of mobile apps in health interventions: a systematic review of the literature. JMIR Mhealth Uhealth 2015 Feb 26;3(1):e20 [FREE Full text] [doi: 10.2196/mhealth.3335] [Medline: 25803705]

21. Coulon SM, Monroe CM, West DS. A systematic, multi-domain review of mobile smartphone apps for evidence-based stress management. Am J Prev Med 2016 Jul;51(1):95-105. [doi: 10.1016/j.amepre.2016.01.026] [Medline: 26993534]

22. Girault A, Ferrua M, Lalloué B, Sicotte C, Fourcade A, Yatim F, et al. Internet-based technologies to improve cancer care coordination: current use and attitudes among cancer patients. Eur J Cancer 2015 Mar;51(4):551-557. [doi: 10.1016/j.ejca.2014.12.001] [Medline: 25661828]

23. Drewes C, Kirkovits T, Schiltz D, Schinkoethe T, Haidinger R, Goldmann-Posch U, et al. EHealth acceptance and new media preferences for therapy assistance among breast cancer patients. JMIR Cancer 2016 Sep 14;2(2):e13 [FREE Full text] [doi: $\underline{10.2196 / \text { cancer.5711] [Medline: 28410189] }}$

24. Klasnja P, Hartzler A, Powell C, Phan G, Pratt W. Health weaver mobile: designing a mobile tool for managing personal health information during cancer care. AMIA Annu Symp Proc 2010 Nov 13;2010:392-396 [FREE Full text] [Medline: 21347007]

25. Trinh L, Arbour-Nicitopoulos KP, Sabiston CM, Alibhai SM, Jones JM, Berry SR, et al. A qualitative study exploring the perceptions of sedentary behavior in prostate cancer survivors receiving androgen-deprivation therapy. Oncol Nurs Forum 2015 Jul;42(4):398-406. [doi: 10.1188/15.ONF.398-406] [Medline: 26148319]

26. Odeh B, Kayyali R, Nabhani-Gebara S, Philip N. Optimizing cancer care through mobile health. Support Care Cancer 2015 Jul;23(7):2183-2188. [doi: 10.1007/s00520-015-2627-7] [Medline: 25649121]

27. Firth J, Torous J. Smartphone apps for schizophrenia: a systematic review. JMIR Mhealth Uhealth 2015 Nov 06;3(4):e102 [FREE Full text] [doi: 10.2196/mhealth.4930] [Medline: 26546039]

28. Moher D, Liberati A, Tetzlaff J, Altman DG, PRISMA Group. Preferred reporting items for systematic reviews and meta-analyses: the PRISMA statement. PLoS Med 2009 Jul 21;6(7):e1000097 [FREE Full text] [doi: 10.1371/journal.pmed.1000097] [Medline: 19621072]

29. Egbring M, Far E, Roos M, Dietrich M, Brauchbar M, Kullak-Ublick GA, et al. A mobile app to stabilize daily functional activity of breast cancer patients in collaboration with the physician: a randomized controlled clinical trial. J Med Internet Res 2016 Sep 06;18(9):e238 [FREE Full text] [doi: 10.2196/jmir.6414] [Medline: 27601354]

30. Foley NM, O'Connell EP, Lehane EA, Livingstone V, Maher B, Kaimkhani S, et al. PATI—patient accessed tailored information: a pilot study to evaluate the effect on preoperative breast cancer patients of information delivered via a mobile application. Breast 2016 Dec;30:54-58. [doi: 10.1016/j.breast.2016.08.012] [Medline: 27611236] 
31. Fromme EK, Kenworthy-Heinige T, Hribar M. Developing an easy-to-use tablet computer application for assessing patient-reported outcomes in patients with cancer. Support Care Cancer 2011 Jun;19(6):815-822 [FRE Full text] [doi: 10.1007/s00520-010-0905-y] [Medline: 20512360]

32. Fu MR, Axelrod D, Guth AA, Rampertaap K, El-Shammaa N, Hiotis K, et al. mHealth self-care interventions: managing symptoms following breast cancer treatment. Mhealth 2016 Jul;2 [FREE Full text] [doi: 10.21037/mhealth.2016.07.03] [Medline: 27493951]

33. Hyun-Ju J, Bok-Hee C. Efficacy of an application-based recurrence prevention program for breast cancer survivors: a pilot study. Indian J Sci Technol 2016;9(46). [doi: 10.17485/ijst/2016/v9i46/107172]

34. Kearney N, McCann L, Norrie J, Taylor L, Gray P, McGee-Lennon M, et al. Evaluation of a mobile phone-based, advanced symptom management system (ASyMS) in the management of chemotherapy-related toxicity. Support Care Cancer 2009 Apr;17(4):437-444. [doi: 10.1007/s00520-008-0515-0] [Medline: 18953579]

35. Matthew AG, Currie KL, Irvine J, Ritvo P, Santa MD, Jamnicky L, et al. Serial personal digital assistant data capture of health-related quality of life: a randomized controlled trial in a prostate cancer clinic. Health Qual Life Outcomes 2007 Jul 06;5:38 [FREE Full text] [doi: 10.1186/1477-7525-5-38] [Medline: 17617906]

36. Quintiliani LM, Mann DM, Puputti M, Quinn E, Bowen DJ. Pilot and feasibility test of a mobile health-supported behavioral counseling intervention for weight management among breast cancer survivors. JMIR Cancer 2016 May 09;2(1):e4 [FREE Full text] [doi: 10.2196/cancer.5305] [Medline: 28410174]

37. Skolarus TA, Holmes-Rovner M, Hawley ST, Dunn RL, Barr KLC, Willard NR, et al. Monitoring quality of life among prostate cancer survivors: the feasibility of automated telephone assessment. Urology 2012 Nov;80(5):1021-1026. [doi: 10.1016/j.urology.2012.07.038] [Medline: 22990056]

38. Somers TJ, Abernethy AP, Edmond SN, Kelleher SA, Wren AA, Samsa GP, et al. A pilot study of a mobile health pain coping skills training protocol for patients with persistent cancer pain. J Pain Symptom Manage 2015 Oct;50(4):553-558 [FREE Full text] [doi: 10.1016/j.jpainsymman.2015.04.013] [Medline: 26025279]

39. Sundberg K, Eklöf AL, Blomberg K, Isaksson A, Wengström Y. Feasibility of an interactive ICT-platform for early assessment and management of patient-reported symptoms during radiotherapy for prostate cancer. Eur J Oncol Nurs 2015 Oct;19(5):523-528. [doi: 10.1016/j.ejon.2015.02.013] [Medline: 25813529]

40. Valle CG, Deal AM, Tate DF. Preventing weight gain in African American breast cancer survivors using smart scales and activity trackers: a randomized controlled pilot study. J Cancer Surviv 2017 Feb;11(1):133-148. [doi:

10.1007/s11764-016-0571-2] [Medline: 27631874]

41. Young-Afat DA, van Gils CH, Bruinvels DJ, van der Pol CC, Witkamp AJ, Sijtsema S, et al. Patients' and health care providers' opinions on a supportive health app during breast cancer treatment: a qualitative evaluation. JMIR Cancer 2016 Jun 07;2(1):e8 [FREE Full text] [doi: 10.2196/cancer.5334] [Medline: 28410170]

42. Kim J, Lim S, Min YH, Shin Y, Lee B, Sohn G, et al. Depression screening using daily mental-health ratings from a smartphone application for breast cancer patients. J Med Internet Res 2016 Aug 04;18(8):e216 [FREE Full text] [doi: 10.2196/jmir.5598] [Medline: 27492880]

43. McCarroll ML, Armbruster S, Pohle-Krauza RJ, Lyzen AM, Min S, Nash DW, et al. Feasibility of a lifestyle intervention for overweight/obese endometrial and breast cancer survivors using an interactive mobile application. Gynecol Oncol 2015 Jun;137(3):508-515. [doi: 10.1016/j.ygyno.2014.12.025] [Medline: 25681782]

44. Min YH, Lee JW, Shin Y, Jo M, Sohn G, Lee J, et al. Daily collection of self-reporting sleep disturbance data via a smartphone app in breast cancer patients receiving chemotherapy: a feasibility study. J Med Internet Res 2014 May 23;16(5):e135 [FREE Full text] [doi: 10.2196/jmir.3421] [Medline: 24860070]

45. Sundberg K, Wengström Y, Blomberg K, Hälleberg-Nyman M, Frank C, Langius-Eklöf A. Early detection and management of symptoms using an interactive smartphone application (Interaktor) during radiotherapy for prostate cancer. Support Care Cancer 2017 Jul;25(7):2195-2204 [FREE Full text] [doi: 10.1007/s00520-017-3625-8] [Medline: 28236145]

46. Uhm KE, Yoo JS, Chung SH, Lee JD, Lee I, Kim JI, et al. Effects of exercise intervention in breast cancer patients: is mobile health (mHealth) with pedometer more effective than conventional program using brochure? Breast Cancer Res Treat 2017 Feb;161(3):443-452. [doi: 10.1007/s10549-016-4065-8] [Medline: 27933450]

47. Landis JR, Koch GG. The measurement of observer agreement for categorical data. Biometrics 1977 Mar;33(1):159-174. [Medline: 843571$]$

48. Cella DF, Tulsky DS, Gray G, Sarafian B, Linn E, Bonomi A, et al. The Functional Assessment of Cancer Therapy scale: development and validation of the general measure. J Clin Oncol 1993 Mar;11(3):570-579. [doi: 10.1200/JCO.1993.11.3.570] [Medline: 8445433]

49. Fallowfield L, Payne H, Jenkins V. Patient-reported outcomes in metastatic castration-resistant prostate cancer. Nat Rev Clin Oncol 2016 Oct;13(10):643-650. [doi: 10.1038/nrclinonc.2016.100] [Medline: 27349193]

50. EuroQol Group. EuroQol—a new facility for the measurement of health-related quality of life. Health Policy 1990 Dec;16(3):199-208. [Medline: 10109801]

51. Aaronson NK, Ahmedzai S, Bergman B, Bullinger M, Cull A, Duez NJ, et al. The European Organization for Research and Treatment of Cancer QLQ-C30: a quality-of-life instrument for use in international clinical trials in oncology. J Natl Cancer Inst 1993 Mar 03;85(5):365-376. [Medline: $\underline{\text { 8433390] }}$ 
52. van Andel G, Bottomley A, Fosså SD, Efficace F, Coens C, Guerif S, et al. An international field study of the EORTC QLQ-PR25: a questionnaire for assessing the health-related quality of life of patients with prostate cancer. Eur J Cancer 2008 Nov;44(16):2418-2424. [doi: 10.1016/j.ejca.2008.07.030] [Medline: 18774706]

53. Sprangers MA, Groenvold M, Arraras JI, Franklin J, te Velde A, Muller M, et al. The European Organization for Research and Treatment of Cancer breast cancer-specific quality-of-life questionnaire module: first results from a three-country field study. J Clin Oncol 1996 Oct;14(10):2756-2768. [doi: 10.1200/JCO.1996.14.10.2756] [Medline: $\underline{8874337]}$

54. Spitzer RL, Kroenke K, Williams JB. Validation and utility of a self-report version of PRIME-MD: the PHQ primary care study. JAMA 1999 Nov 10;282(18):1737-1744. [Medline: 10568646]

55. Kroenke K, Spitzer RL, Williams JB. The PHQ-9: validity of a brief depression severity measure. J Gen Intern Med 2001 Sep;16(9):606-613 [FREE Full text] [Medline: 11556941]

56. Cohen S, Kamarck T, Mermelstein R. A global measure of perceived stress. J Health Soc Behav 1983 Dec;24(4):385-396. [Medline: $\underline{6668417]}$

57. Lyons EJ, Baranowski T, Basen-Engquist KM, Lewis ZH, Swartz MC, Jennings K, et al. Testing the effects of narrative and play on physical activity among breast cancer survivors using mobile apps: study protocol for a randomized controlled trial. BMC Cancer 2016 Mar 09;16:202 [FREE Full text] [doi: 10.1186/s12885-016-2244-y] [Medline: 26960972]

58. Lozano-Lozano M, Martín-Martín L, Galiano-Castillo N, Álvarez-Salvago F, Cantarero-Villanueva I, Fernández-Lao C, et al. Integral strategy to supportive care in breast cancer survivors through occupational therapy and a m-health system: design of a randomized clinical trial. BMC Med Inform Decis Mak 2016 Nov 25;16(1):150 [FREE Full text] [doi: 10.1186/s12911-016-0394-0] [Medline: 27887610]

59. Maguire R, Fox PA, McCann L, Miaskowski C, Kotronoulas G, Miller M, et al. The eSMART study protocol: a randomised controlled trial to evaluate electronic symptom management using the advanced symptom management system (ASyMS) remote technology for patients with cancer. BMJ Open 2017 Jun 06;7(5):e015016 [FREE Full text] [doi: 10.1136/bmjopen-2016-015016] [Medline: 28592577]

\author{
Abbreviations \\ BDI: Beck Depression Inventory \\ CAP: College of American Pathologists \\ CLIA: Clinical Laboratory Improvement Amendments \\ Questionnaire-Core \\ complementary module for breast cancer patients \\ complementary module for prostate cancer patients \\ EQ-5D-3L: EuroQol 5-Dimension questionnaire \\ FACT-G: Functional Assessment of Cancer Therapy-General \\ HRQoL: health-related quality of life \\ mHealth: mobile health \\ PHQ-9: Patient Health Questionnaire-9 \\ PRISMA: Preferred Reporting Items for Systematic Reviews and Meta-Analyses \\ PRO: patient-reported outcome measures \\ PSS: Perceived Stress Scale \\ QoL: quality of life \\ RCT: randomized controlled trial \\ OSR: online store research \\ WEL: Weight Efficacy Lifestyle questionnaire
}

EORTC QLQ-C30: European Organization for Research and Treatment of Cancer Quality of Life

EORTC QLQ-BR23: European Organization for Research and Treatment of Cancer Quality of Life Questionnaire

EORTC QLQ-PR25: European Organization for Research and Treatment of Cancer Quality of Life Questionnaire

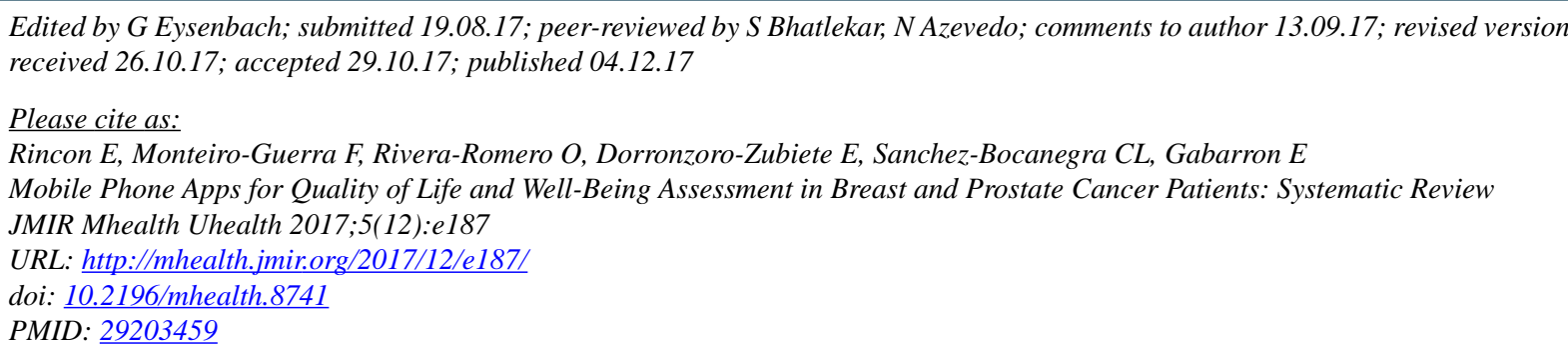


(CEsther Rincon, Francisco Monteiro-Guerra, Octavio Rivera-Romero, Enrique Dorronzoro-Zubiete, Carlos Luis Sanchez-Bocanegra, Elia Gabarron. Originally published in JMIR Mhealth and Uhealth (http://mhealth.jmir.org), 04.12.2017. This is an open-access article distributed under the terms of the Creative Commons Attribution License (https://creativecommons.org/licenses/by/4.0/), which permits unrestricted use, distribution, and reproduction in any medium, provided the original work, first published in JMIR mhealth and uhealth, is properly cited. The complete bibliographic information, a link to the original publication on http://mhealth.jmir.org/, as well as this copyright and license information must be included. 